\title{
ДУПЛЕКСЕР НА СИММЕТРИЧНОЙ ПОЛОСКОВОЙ ЛИНИИ ПЕРЕДАЧИ
}

\author{
А. В. ЗАХАРОВ, С. А. РОЗЕНКО, Л. С. ПИНЧУК \\ Национальный технический университет Украинь \\ «Киевский политехнический институт им. Игоря Сикорского», \\ Украина, Киев, 03056, пр-т Победы 37
}

\begin{abstract}
Аннотация. Приведено построение и осуществлено измерение характеристик малогабаритного дуплексера на симметричной полосковой линии передачи, который содержит гребенчатые фильтры пятого порядка в каналах передачи $T X(2300-2370$ МГц) и приема $R X(2510-2580$ МГц). Дуплексер выполнен на диэлектрическом материале $\mathrm{Al}_{2} \mathrm{O}_{3}$ (alumina, поликор) с высокой теплопроводностью, что позволило использовать рабочую мощность 10 Вт при малых габаритах $57 \times 11,8 \times 4$ мм. Потери в полосах пропускания $T X$ и $R X$ фильтров не превышали 3 дБ при затухании фильтров на частотах соседнего канала не менее 60 дБ. Показано, что выбором ширины металлизированной полоски у основания четвертьволновых резонаторов возможно изменять ширину дуплексера, добиваясь требуемой величины. Используемая в конструкции цепь связи резонаторов с нагрузками позволила получить достаточно высокий уровень изоляции друг от друга каналов $R X$ и $T X$ дуплексера, превышающий 60 дБ. При построении дуплексера учтена конечная толщина внутренних проводников полосковой линии, составляющая 16 мкм, что привело к хорошему соответствию результатов моделирования и измерения.
\end{abstract}

Ключевые слова: дуплексер; гребенчатый фильтр; каналы приема и передачи; уровень развязки; рабочая мощность

\section{ВВЕДЕНИЕ}

Дуплексеры на основе керамических материалов являются неотъемлемой частью мобильных систем связи, в том числе и сотовой телефонии. Они осуществляют разделение сигналов в каналах приема и передачи $[1,2]$, где канал приема обозначают $R X$, а канал передачи $T X$. В каналах $R X$ и $T X$ могут использоваться фильтры верхних и нижних частот, известно также сочетание в дуплексере полосно-пропускающего и полосно-заграждающего фильтров. Наиболее часто в дуплексере объединяют два гребенчатых полосно-пропускающих фильтра. Использование гребенчатой структуры $R X$ и $T X$ фильтров, а также керамики с высокой диэлектрической проницаемо- стью $\varepsilon_{r}$ обеспечивает дуплексерам малые габариты. Дуплексеры выпускаются в больших количествах высокотехнологичными компаниями, такими как Murata (Япония) [3], CTS Corporation (США) [4].

Известно различное конструктивное выполнение дуплексеров. Особое распространение получили дуплексеры на коаксиальных диэлектрических резонаторах [3] и в моноблочном исполнении с рядом резонансных отверстий $[4,5]$. Использование микрополосковых полосно-пропускающих фильтров на подложках с высоким значением $\varepsilon_{r}$ [6] позволило создать малогабаритные микрополосковые дуплексеры [7], размеры которых соизмеримы с размерами дуплексеров на коаксиальных диэлектрических резонаторах. Последние из из- 


\section{БИБЛИОГРАФИЧЕСКИЙ СПИСОК}

1. Hunter, I. Theory and design of microwave filters. The Institution of Engineering and Technology, London, 2006. 368 p. 
2. Makimoto, M.; Yamashita, S. Microwave Resonators and Filters for Wireless Communication. Springer Science \& Business Media, 2001.

3. http://www.murata.com.

4. http://www.ctscorp.com.

5. Fukasawa, A. Analysis and composition of a new microwave filter configuration with inhomogeneous dielectric medium. IEEE Trans. Microwave Theory Tech., Vol. 30, No. 9, P. 1367-1375, 1982. DOI: $\underline{10.1109 /}$ TMTT.1982.1131262.

6. Захаров, А.В.; Розенко, С. А.; Захарова, Н. А. Микрополосковые полосно-пропускающие фильтры на подложках с высокой диэлектрической проницаемостью. Радиотехника и электроника, Т. 57, № 3, С. 372-382, 2012. URI: https://elibrary.ru/item.asp?id=1764 7462.

7. Захаров, А. В.; Розенко, С. А. Дуплексер на основе микрополосковых фильтров, использующих подложки с высокой диэлектрической проницаемостью. Радиотехника и электроника, Т. 57, № 6, С. 713-720, 2012. URI: https://elibrary.ru/item.asp?id=17726257.

8. Nwajana, A. O.; Yeo, K. S. K. Microwave diplexer purely based on direct synchronous and asynchronous coupling. Radioengineering, Vol. 25, No. 2, P. 247-252, 2016. DOI: $10.13164 /$ re.2016.0247.

9. Ndujiuba, Charles U.; John, Samuel N.; Bello, Taofeek O. Design of duplexers for microwave communication systems using open-loop square microstrip resonators. Int. J. Electromagnetics Applications, No. 6(1), P. 7-12, 2016. DOI: 10.5923/j.ijea.20160601.02.

10. Lee, J.-H.; Kidera, N.; DeJean, G.; Pinel, S.; Laskar, J.; Tentzeris, M. M. A V-band front-end with 3-D integrated cavity filters/duplexers and antenna in LTCC technologies. IEEE Trans. Microwave Theory Tech., Vol. 54, No. 7, P. 2925-2936, 2006. DOI: 10.1109/TMTT. 2006.877440 .

11. Захаров, А. В.; Ильченко, М.Е. Тонкие полосно-пропускающие фильтры, содержащие отрезки симметричных полосковых линий передачи. Радиотехника и электроника, Т. 58, № 7, С. 716-725, 2013. DOI: 10.7868/S0033849413060156.

12. Захаров, А.В. Полосковые гребенчатые фильтры на керамических материалах с высокой ди- электрической проницаемостью. Радиотехника $u$ электроника, Т. 58, № 3, C. 300-308, 2013. DOI: 10.7868/S0033849413030145.

13. Bolljahn, J. T.; Matthaei, G. L. A study of the phase and filter properties of arrays of parallel conductors between ground planes. Proc. IRE, Vol. 50, No. 3, P. 299-311, 1962. DOI: 10.1109/JRPROC.1962.288322.

14. Маттей, Г. Л.; Янг, Л.; Джонс, Е.М. Т. Фильтры СВЧ, согласующие иепи и ичепи связи. М.: Связь, 1971.

15. Dworsky, L. N. Stripline filters - An overview. Proc. of 37th Annual Symp. of Frequency Control, 1-3 June 1983, Philadelphia, Pennsylvania, USA. IEEE, 1983, pp. 387-393. DOI: 10.1109/FREQ.1983.200697.

16. Hong, J.-S. Microstrip Filters for RF/Microwave Application, 2nd ed. N.Y.: Wiley, 2011.

17. Захаров, А. В.; Ильченко, М.Е.; Пинчук, Л. С. Зависимость коэффициента связи между четвертьволновыми резонаторами от параметров гребенчатых полосковых фильтров. Известия вузов. Радиоэлектроника, Т. 58, № 6, С. 52-60, 2015. URI: http://radio. kpi.ua/article/view/S0021347015060060.

18. Захаров, А.В.; Ильченко, М.Е.Полосно-пропускающие фильтры решетчатого типа на основе полуволновых резонаторов из отрезков симметричных полосковых линий передачи. Радиотехника и электроника, Т. 60, № 7, C. 759-765, 2015. DOI: $\underline{10.7868 /}$ S0033849415060182.

19. Захаров, А.В.; Ильченко, М.Е.; Карнаух, В.Я.; Пинчук, Л.С.Полосковые полосно-пропускающие фильтры со ступенчатыми резонаторами. Известия вузов. Радиоэлектроника, Т. 54, № 3, С. 56-63, 2011. URI: http://radio.kpi.ua/article/view/S0021347011 030071 .

20. Справочник по расчету и конструированию СВЧ полосковых устройств. Под ред. В.И. Вольмана. М.: Радио и связь, 1982.

21. Справочник по элементам полосковой техники. Под ред. А. Л.Фельдштейна. М.: Связь, 1979.

22. http://www.ceramics.sp.ru. 